\title{
Journal of Gynecology and Neonatal Biology
}

\section{Participation in Cancer Biobanking at a German Department for Obstetrics and Gynecology}

\author{
Juliane Nees', Sylwia Wejchert ${ }^{1}$, Thomas Bruckner², Christof Sohn', Joachim Rom ${ }^{1}$, Sarah Schott ${ }^{1,3^{*}}$ \\ ${ }^{1}$ University Hospital Heidelberg, Department of Gynecology and Obstetrics, Im Neuenheimer Feld 440, 69120 Heidelberg, \\ Germany \\ ${ }^{2}$ Institute of Medical Biometry and Informatics, University of Heidelberg, Im Neuenheimer Feld 130.3, 69120 Heidelberg, \\ Germany \\ ${ }^{3}$ DKTK, German Cancer Consortium DKFZ Heidelberg, Im Neuenheimer Feld 280, 69120 Heidelberg, Germany
}

*Corresponding Author: Prof. Dr. Sarah Schott, MD, PhD, University Hospital Heidelberg, Department of Gynecology and Obstetrics, Im Neuenheimer Feld, Heidelberg, Germany, DKTK, DKFZ Heidelberg, Im Neuenheimer Feld,Heidelberg, Germany, Tel: +496221 560/Fax +496221 5633997; E-mail: sarah.schott@med.uni-heidelberg.de

\begin{abstract}
Biobanking is an essential requirement for the success of translational oncology. The demand for biomaterials for several studies is often large and results in dissatisfaction. Confirmed consent remains the key issue to providing biobanking material. In this study, we evaluated the willingness of patients undergoing surgery to participate in biobanking studies and reasons for exclusion. The aim of this study is to outline the pitfalls in terms of physician attempts to gain biomaterial. Results will contribute to optimized recruitment and an understanding of patient reservations.

Patients attending elective surgery were evaluated April - June 2016 for potential inclusion in several biobanking studies and were checked in respect to tumor size and origin. During their informed consent consultation, 527 patients were screened. The median age was 48 years. Of the 527 patients, 120 were eligible for biobanking. The reasons for exclusion were: 264 patients with no oncological surgery, 46 with a tumor too small for biobanking, 19 with second surgery, and 78 patients with a tumor not suitable for any biobanking study. Of the 120 included patients, 92 suffered from breast cancer, 21 had ovarian cancer, one had carcinoma of the cervix, and another six had endometrial cancers. 29 of the patients had undergone neoadjuvant chemotherapy. Of the 120 patients, 109 agreed to participate in biobanking. Mostly, patients are motivated to contribute to biobanking. Neoadjuvante chemotherapy limits participation in biobanking due to missing residual tumor mass. The improvement of systemic treatment in terms of breast cancer might need to focus on core cut biopsies.
\end{abstract}

Received date: November 28, 2017

Accepted date: January 8, 2018

Published date: January 11, 2018

Citation: Ness, J., et al. Participation in Cancer Biobanking at a German Department for Obstetrics and Gynecology (2018) J Gynecol Neonatal Biol 4(1): 13.

DOI: $10.15436 / 2380-5595.18 .1742$

Kyewords: Biobanking, Pitfalls, Translational oncology

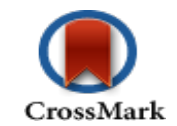

\section{Introduction}

Biobanking is an essential requirement for the success of translational oncology and an important tool to improve medical treatments ${ }^{[1]}$. The last decades have focused on the development of individual cancer care, aiming for a rapid translation from bench to bedside in order to improve cancer diagnostics as well as therapeutic strategies. Modern investigations using omics-technologies are striving to establish personalized medicine. Standardized sampling and tissue processing are essential for sensitive high-end analyses. Ideally, fresh tumor tissue and processed blood samples would be obtained so that clinical pathways can be analyzed. Additionally, tumor markers, laboratory kits, and suspicious genetic hits need validation in biomaterial ${ }^{[2]}$.

Clinical physicians and researchers often face pitfalls in their surroundings, leading to access limitations, for example to the patients' materials in general or an insufficient amount of patient-derived material for e.g. cell cultures. The simultaneous demand for biomaterials across several studies can be high and supplies limited, resulting in dissatisfaction for all participating parties. Motivational factors and the high work load are two of the major issues that can lead to a loss in recruitment. Other

Copyrights: (C) 2018 Schott, S. This is an Open access article distributed under the terms of Creative Commons Attribution 4.0 International License. 
studies have shown that patients' willingness to participate in biobanking is generally rather high ${ }^{[3,4]}$. This study evaluates patients' willingness and eligibility to participate in tissue banking. To our knowledge this study is the first to outline the work flow and its pitfalls from a clinical perspective.

\section{Methods}

This is an explorative, monocenter study at the Heidelberg University Hospital, Department of Gynecology and Obstetrics, which screened patients in respect to eligibility for biobanking during preoperative consultations between April and June 2016. If eligible, the patients were briefed on the opportunities of biobanking for current ongoing local studies as well as general biobanking for the National Center for Tumor Diseases (NCT) Heidelberg bank. It was explained that the studies did not have any impact on their current situation but might make an essential contribution to future diagnostic and therapeutic approaches. Afterwards, they were required to provide written informed consent in order to participate during their preoperative informed consent conversation. The patients were assessed by four different medical doctors. In the event that they refused to participate in biobanking, they were questioned in a standardized manner regarding the reason. The reasons were classified as psychological and/or physical distress or weakness, mistrust/ fear of studies, or a general refusal for studies.

\section{Participant eligibility and evaluation}

All patients suffering from breast cancer $>$ CT1b at the time of surgery with or without neoadjuvant chemotherapy as well as ovarian, cervical, or endometrial cancer at any stage were eligible to participate in biobanking. The reasons for exclusion were categorized as follows; no oncological surgery, tumor size too small, other location, and second tumor resection. Data were documented in MS Excel. The data analysis was performed using SAS version 9.4 WIN. Results were summarized using absolute and relative frequencies when reporting categorical data and mean and standard deviation when reporting continuous data.

\section{Results}

Between April and June 2016, 527 patients were screened for the option of biobanking during their first preoperative consultation. There were 526 women and one man. The average age was 49 years with a standard deviation of 12 years. Out of the 527 patients, 120 were potentially eligible for biobanking due to local studies. The reasons for exclusion were mainly: 264 no oncological surgery (65\%) was planned; 46 had a tumor that was too small for biobanking (11\%); 78 patients had a tumor without current study opportunities (19\%) and 19 with a second tumor resection $(5 \%)$.

Among the 120 participants, 92 (77\%) suffered from breast cancer, $21(18 \%)$ from ovarian cancer, $1(0.8 \%)$ from cervical cancer, and 6 (5\%) from endometrial cancer. $24 \%$ of the breast cancer patients had undergone systemic neoadjuvant treatment. Of the 120 patients, 109 (91\%) agreed to biobanking, and $11(9 \%)$ decided not to participate due to physical and psychological strain, a fear of clinical studies, or/ a general refusal of clinical studies.

\section{Discussion}

This study addresses the question of biobanking participation. Our results show that in general few patients $(22 \%)$ undergoing for gynecological surgery in our Department of Gynecology and Obstetrics are candidates to participate in ongoing clinical biobanking trials. In respect to recent studies for translational oncology evaluating certain biomarkers, it is the aim to collect general biomaterials not only for ongoing studies, but upcoming studies as well. Therefore, it would be advisable to not only include oncological patients, but also women with benign conditions these samples will open options to compare healthy tissue to malignancies. In times of personal medicine, it is essential to have larger samples sizes to precisely address scientific questions in sub- cohorts. Therefore, broader biobanking would be very desirable. For example, this is the aim of the biobanking practices of the National Center of Tumor Diseases (NCT), which collects biomaterial for collaboration within the DKTK network. Furthermore, our outcomes illustrate that patients' willingness to participate in biobanking is high (90\%). Therefore, broad biobanking should generally be possible. Similar results were presented by Byrant et al in 2015 in their study with 224 oncological patients, $84 \%$ of whom were willing to donate tissue ${ }^{[3]}$. Portery et al also found high motivation to give biological samples to a biobank in their study with 145 subjects, of whom $86 \%$ were inclined to donate ${ }^{[4]}$. A study by Rahm et al did not show such a high willingness for biobanking; only $69 \%$ of their 203 participants were inclined to give a blood sample for biobanking ${ }^{[5]}$. One reason for this difference might be that in our study the patients had no additional examination when they joined biobanking, whereas in Rahm's study, they had to do an extra blood draw. This might indicate that simple access and obtaining material during operations, when there is no additional harm for the patient, could benefit biobanking. Therefore, it is the doctor's responsibility to outline the impact of biobanking on research and of everyone's contribution on future diagnosis and therapeutic strategies in a way that underlines that there is no extra impact for the patient. A personal benefit for patients or their relatives might also be an incentive to participate. This might be an argument for participating that would be considered by the patients. Doctors' assistants or study nurses could also contribute to simplify the process. Additional campaigns and perhaps flyers or homepage announcements might also contribute to make biobanking an established practice.

Reasons for refusal of the 11 patients who did not want to join biobanking included physical strain, a fear of clinical studies, and a general refusal of clinical studies. Rahm et al described that that confidentiality and security fears were the most common reasons for not participating in their study. Furthermore, Portery et al. found that the willingness to donate biological samples depends on the patients' level of education: the higher the level, the higher the percentage of the patients willing to donate ${ }^{[4,5]}$. This small sample size does not allow any further conclusions. However, the reasons stated by our patients and the findings seen in the literature support the recommendations made above; namely that strategies are needed to explain that biobanking studies on are not to be feared nor do they impact a patient's treatment at all. The second most common reason for exclusion among cancer patients is a small tumor size when they come for surgery. One reason in the breast cancer setting 
could be the increasing number of patients receiving neoadjuvant chemotherapy resulting in a partial or complete response, such as the GeparSixto study we recently participated in where we achieved a complete pathological response in $36.9-43.7 \%$ of the patients with neoadjuvant chemotherapy ${ }^{[6]}$; in this setting, biobanking when surgery eventually is performed is impossible, as is obtaining representative samples for both the pathological evaluation as well as for research purposes. Another reason for the small tumor size when patients come for surgery is the screening detection rate of small tumors or precursor lesions. These underlying reasons are responsible for the rather high number of cases biobanking failed initially. One possibility to obtain primary tissue samples might be during core cut biopsy instead. However, the timing of core cut biopsy is normally the first confrontation with the possibility of a malignant disease and is mostly a stressful situation for patients. This is not the optimal setting to confront the patients with study issues, and it might be viewed as an extra burden and unnecessary pain during the operation, which is not the case.

In addition, the option of general biobanking to gain material for upcoming studies should be considered in order to not burden patients if an anonymous tumor donation with regular diagnostic pathology work is the study purpose. Otherwise it might be more sensitive to outline and explain the study options prior to a doctor's visit, i.e. in the waiting room, online, or through cancer nurses, in order to not additionally stress patients. There is a need to improve the general understanding of what "study" means in terms of biobanking as it mostly contributes to cancer care that does not impact the patient personally but may have a huge impact on future diagnosis and therapy.

Furthermore, physicians' awareness plays a key role in recruiting patients. The investigator tasked with recruiting the patients preoperatively might not be the primary physician or study leads. Therefore, it is very important to improve the awareness of each ongoing study. As Caixeiro et al showed a lack of support from physicians is a limiting factor for the establishment of biobanks; however, they have issued a general declaration of support ${ }^{[1]}$.

In conclusion, we found that most patients and physicians are willing to support the establishment of biobanking, which is a key ingredient for cancer research. Because patients are so rarely eligible for biobanking, it is essential that physicians be aware of it, though patients should never be burdened due to studies in emotionally challenging situations, such as during the initial cancer diagnosis. It may be wise to obtain biobank material before neoadjuvant chemotherapy commences and approach every patient regarding tissue donation even if no specific study is ongoing at the time of surgery.
Acknowledgement: The authors thank Katherine Taylor for critical reading

\section{Conflict of interest}

The authors declare that they have no conflict of interest

\section{Informed consent}

Informed consent was obtained from all participants included in biobanking studies

\section{References}

1) Caixeiro, N.J., Byun, H.L., Descallar, J., et al. "Health professionals' opinions on supporting a cancer biobank: identification of barriers to combat biobanking pitfalls. (2016) Eur J Hum Genet 24(5): 626-632. Pubmed | Crossref | Others

2) Wheelock, Å.M., Paulson, L., Litton, J.E., et al. "The EuPA Biobank Initiative: Meeting the future challenges of biobanking in proteomics \& systems medicine.”(2015) J Proteomics 127(Pt B): 414-416.

Pubmed |Crossref| Others

3) Bryant, J., Sanson-Fisher, R., Fradgley, E., et al. Oncology patients overwhelmingly support tissue banking. (2015) BMC cancer 15(1): 413.

Pubmed | Crossref | Others

4) Porteri, C., Pasqualetti, P., Togni, E., et al. Public's attitudes on participation in a biobank for research: an Italian survey. (2014) BMC Med Ethics 15: 81.

Pubmed | Crossref | Others

5) Rahm, A.K., Wrenn, M., Carroll, N.M., et al. Biobanking for research: a survey of patient population attitudes and understanding. (2013) J Community Genet 4(4): 445-450.

Pubmed | Crossref|Others

6) Von, M.G., Schneeweiss, A., Loibl, S., et al. Neoadjuvant carboplatin in patients with triple-negative and HER2-positive early breast cancer (GeparSixto; GBG 66): a randomised phase 2 trial. (2014) Lancet Oncol 15(7): 747-56.

Pubmed | Crossref | Others
Ommega Online Publisher

Journal of Gynecology and Neonatal Biology

Short Title : J Gynecol Neonatal Biol
ISSN: 2380-5595

E-mail : gynecology@ommegaonline.org

website: www.ommegaonline.org 